\title{
An explicit Wave Based model as alternative to the DtN map for solving unbounded Helmholtz problems with the Finite Element method
}

\author{
Bart Bergen $^{\mathrm{a}}$, Elke Deckers ${ }^{\mathrm{a}, *}$, Bert Van Genechten $^{\mathrm{a}}$, Dirk Vandepitte ${ }^{\mathrm{a}}$, \\ Wim Desmet ${ }^{\mathrm{a}}$ \\ ${ }^{a} K U$ Leuven, Department of Mechanical Engineering, division PMA, \\ Celestijnenlaan 300B - box 2420, B-3001 Leuven, Belgium
}

\begin{abstract}
When using a domain-based method for solving problems in unbounded domains, the computational domain has to be truncated to a bounded region. On the truncation, suitable conditions must be imposed to ensure the solution of the truncated problem is equivalent to the solution of the unbounded problem. This paper proposes to derive these conditions based on an explicit model of the unbounded domain exterior to the truncation. The Wave Based Method (WBM), a Trefftz-based prediction technique for solving Helmholtz problems, is used to construct this unbounded model. The WBM has proven to be an efficient alternative for element based techniques and therefore can be applied towards mid-frequency problems. The obtained method is compared to the DtN FE method, where it is shown that the obtained accuracy is equivalent, but the proposed hybrid FE-WB framework allows for a more efficient construction and solution of the model.
\end{abstract}

\footnotetext{
*Corresponding author

Email address: elke.deckers@mech.kuleuven.be (Elke Deckers)
} 
Keywords:

Helmholtz problems, unbounded problems, Finite Element Method, Wave Based Method, Dirichlet-to-Neumann map

\section{Introduction}

The solution of Helmholtz problems involving unbounded problem domains has been the subject of substantial research effort. When solving this problem with a domain-based method, the problem domain is first truncated to a bounded region, to make the problem amenable to practical implementation. This truncation is artificial, however, and care must be taken to impose appropriate conditions on this truncation, such that the obtained bounded problem is well-posed and its solution matches the solution of the unbounded problem with the desired accuracy. Several methods have been proposed and examined to this end [1]: absorbing boundary conditions (ABCs, either local or non-local), perfectly matched layers (PML) and Infinite Elements (IE).

The ABCs comprise a wide array of different approaches. Bayliss et al. [2] and Enquist et al. [3] proposed a formulation for local ABCs of arbitrary order. The use of higher-order derivatives in these formulations, however, limits the practical applicability in Finite Element (FE) schemes. Several techniques have been proposed to relax this limitation, usually based on the introduction of auxiliary variables to eliminate the higher-order derivatives $[4,5]$. The Dirichlet-to-Neumann (DtN) map $[6,7,8]$ is the most noteworthy non-local condition. This method uses a harmonic expansion of an analytical solution of the exterior problem to relate Dirichlet data to Neumann data on the truncation. The method offers excellent accuracy and stability, but 
is often computationally expensive: the construction requires multiple integrations over the truncation, and the resulting system contains a dense and complex block, deteriorating the efficiency of dedicated FE solvers.

The PML concept, originally proposed by Berenger [9], consists of introducing an artificial layer of elements exterior to the bounded problem. An adapted formulation used in these elements damps out any incoming waves, such that they are absorbed before they reach the outer layer of the PML. As a standard finite element discretisation can be used in the absorbing layer, the sparsity of the resulting system is preserved; it contains nevertheless complex coefficients due to the absorbing formulation. The choice of a good (optimal) absorbing function, relative to the layout of the problem and PML, is subject of substantial research $[10,11]$.

Infinite elements [12] take a somewhat different approach by proposing an explicit model for the unbounded field exterior to the truncation. To fit in with the FE discretisation, a new element is proposed which extends to infinity away from the truncation. The basis used in these elements is selected to be compatible with the FE formulation on the truncation (angular functions), while the radial functions are defined to represent the outgoing wave behaviour. Different formulations are proposed mainly by Burnett [13] and Astley [14], where a trade-off has to be made between near-field accuracy, far-field accuracy and stability of the formulation.

Recently, a Wave Based Method (WBM) [15, 16, 17] was proposed as an alternative technique for the solution of Helmholtz problems. It follows a Trefftz approach [18], using an expansion of basis functions which exactly satisfy the governing dynamic equations to describe the dynamic field. In 
this way, no approximation error is made inside the domain. However, the wave functions violate the boundary and continuity conditions. Enforcing the associated residual errors to zero in a weighted residual scheme yields a small system of algebraic equations, which can be solved for the unknown contribution factors in the expansion. The size of the numerical models and the associated computational resources are substantially lower as compared to element based methods. Because of the enhanced convergence properties, the WBM has proven to be applicable for low- as well as mid-frequency acoustic problems. With a proper selection of the wave functions in the basis, the WBM can be efficiently used to model also problems in unbounded and semi-unbounded domains [19, 20, 21, 22, 23].

This paper discusses the use of such an unbounded Wave Based (WB) model exterior to the truncation of a FE model of an unbounded problem. As the WBM contains an intrinsic formulation for unbounded problems, coupling the FEM with such an unbounded WB model mitigates the need for an implicit absorbing boundary condition. Instead, this condition is replaced by an explicit WB model of the unbounded region exterior to the FE discretisation. The basis functions used in such an unbounded WB model are solutions of the problem exterior to a circle (for two-dimensional problems); the same solution is used to derive the DtN map for this problem. Using the framework of a hybrid WB-FE method, derived earlier for bounded problems [24], an appropriate condition on the FE truncation can be obtained. The proposed method shares the accuracy and the global nature with the DtN FE method. It is shown, however, that the hybrid FE-WB framework allows a more efficient construction of the non-reflecting condition by introducing 
the wave function participation factors as independent degrees of freedom (DOFs) in a coupled system. Using a three-step solution procedure [25], the real and sparse nature of the FE system can be retained, allowing efficient solution with dedicated solvers.

This paper is organised as follows. Section 2 gives the mathematical problem description of a 2D unbounded acoustic Helmholtz problem and the associated FE formulation. As the problem domain is unbounded, a truncation boundary with a suitable boundary condition needs to be introduced. Section 3 describes the DtN map to provide the needed boundary conditions. The novel alternative solution, using an explicit unbounded WB model to account for the unbounded part of the domain exterior to the truncation is discussed in section 4 . The properties of both approaches are compared in section 5. Two numerical verification cases in section 6 show the computational advantage of the explicit unbounded WBM as compared to the use of the DtN map. The paper ends with summarising the main contributions and conclusions

\section{Problem description and FE formulation}

Consider a general two-dimensional unbounded acoustic Helmholtz problem as shown in figure 1. The fluid is assumed to be inviscid with ambient fluid density $\rho_{0}$ and speed of sound $c$. The solution $p(\boldsymbol{r})$ is defined by:

$$
\nabla^{2} p(\boldsymbol{r})+k^{2} p(\boldsymbol{r})=\mathcal{F}(\boldsymbol{r})
$$

with $k=\omega / c$ the acoustic wave number and $\omega$ the circular frequency. $\nabla^{2}$ denotes the Laplacian operator, $\mathcal{F}(\boldsymbol{r})$ describes the sources acting on the 


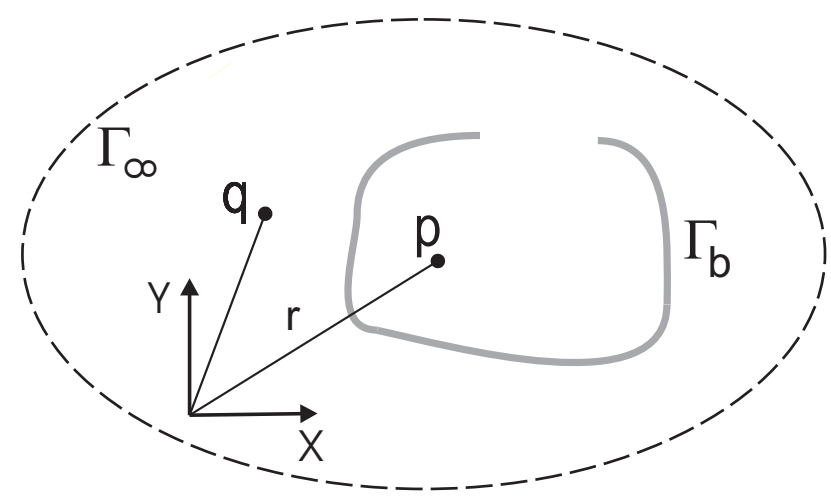

Figure 1: Two-dimensional unbounded Helmholtz problem

computational domain. The problem boundary $\Gamma$ constitutes 2 parts: the finite part of the boundary, $\Gamma_{b}$, and the ficticious boundary at infinity, $\Gamma_{\infty}$. On the finite part of the boundary, a general boundary condition is imposed:

$$
\mathcal{B}(p(\boldsymbol{r}))=0 \text { on } \Gamma_{b}
$$

with $\mathcal{B}$ a boundary condition differential operator. At the boundary at infinity $\Gamma_{\infty}$ the Sommerfeld radiation condition [26] for outgoing waves should be satisfied. This condition ensures that no acoustic energy is reflected at infinity and is expressed as

$$
\lim _{|\boldsymbol{r}| \rightarrow \infty}\left(\sqrt{|\boldsymbol{r}|}\left(\frac{\partial p(\boldsymbol{r})}{\partial|\boldsymbol{r}|}+j k p(\boldsymbol{r})\right)\right)=0
$$

Solution of the Helmholtz equation (1) together with the associated boundary conditions (2)-(3) yields a unique dynamic field $p(\boldsymbol{r})$.

\subsection{FE model}

The problem domain is in a first step divided into a bounded and an unbounded part by introducing the truncation $\Gamma_{t}$ as shown in figure 2 . The 
FEM discretises the bounded part of the problem $\Omega_{b}$ between the boundaries $\Gamma_{b}$ and $\Gamma_{t}$. Within this region, the solution field, in this case the pressure $p(\boldsymbol{r})$ is described using the polynomial FE shape functions $N_{p}(\boldsymbol{r})$ :

$$
p(\boldsymbol{r}) \simeq u(\boldsymbol{r})=\sum_{p=1}^{n_{f e}} N_{p}(\boldsymbol{r}) d_{p},
$$

with $d_{p}$ the nodal DOFs and $n_{f e}$ the number of nodes in the model. On the

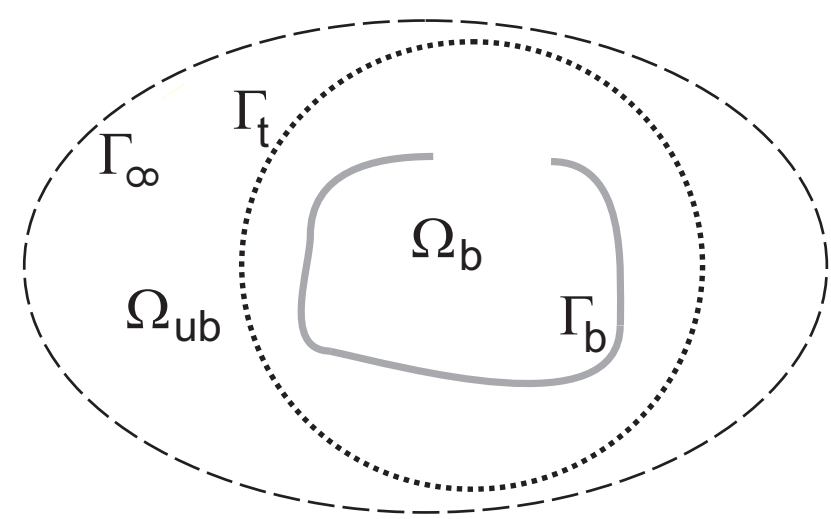

Figure 2: Truncation of the problem for the FEM

finite part of the boundary $\Gamma_{b}$, a general boundary condition is imposed:

$$
\frac{\partial u}{\partial n}(\boldsymbol{r})+\mathcal{B}(u(\boldsymbol{r}))=0 \text { on } \Gamma_{b},
$$

with $\boldsymbol{n}$ the local normal on boundary pointing outwards of $\Omega_{b}$. The boundary $\Gamma_{t}$ is used to truncate the FE model to a bounded region. To compensate for this truncation, a suitable condition should be imposed along this boundary, simulating the continuation of the actual field outside the FE discretisation. This formulation is contained in the operator $\mathcal{T}$ :

$$
\frac{\partial u}{\partial n}(\boldsymbol{r})-\mathcal{T}(u(\boldsymbol{r}))=0 \text { on } \Gamma_{t}
$$


Compliance with the Helmholtz equation (1) and the boundary (5) and continuity (6) conditions on $\Gamma_{b}$ and $\Gamma_{t}$, respectively, is enforced in a weighted residual formulation, yielding the weak form:

$$
\int_{\Omega_{b}}\left(\nabla u \cdot \nabla v-k^{2} u v+v \mathcal{F}\right) \mathrm{d} \Omega_{b}+\int_{\Gamma_{b}} v \mathcal{B}(u) \mathrm{d} \Gamma_{b}-\int_{\Gamma_{t}} v \mathcal{T}(u) \mathrm{d} \Gamma_{t}=0 .
$$

Using a Galerkin approach, the test function $v$ is expanded using the same shape functions that are applied to approximate the dynamic field $u$.

The two next sections describe two distinct ways to construct $\mathcal{T}$ : (i) the DtN map is discussed, and (ii) an alternative explicit unbounded WB model is introduced.

\section{Construction of $\mathcal{T}$ : DtN FE model}

The Dirichlet-to-Neumann (DtN) map, a non-local absorbing boundary condition, is a technique to enable the application of the FEM to unbounded problems. The DtN map [6] constructs a mapping of Dirichlet (pressure) data on the truncation $\Gamma_{t}$ to Neumann (velocity) data on the same boundary, using an analytical model for the dynamic field exterior to $\Gamma_{t}$. In general such a map takes the form:

$$
\frac{\partial u}{\partial n}=M u, \text { on } \Gamma_{t},
$$

where $n$ is the unit normal on $\Gamma_{t}$ pointing outwards. The operator $M$ is the mapping operator, describing the relation between the Dirichlet and Neumann data. The DtN map proposed by Keller and Givoli [6] uses a multipole expansion to describe the field in $\Omega_{u b}$. This model is not used explicitly to describe the field, but instead condensed to a relation between pressure and 
velocity on $\Gamma_{t}$ the so-called DtN map:

$$
M u=\frac{1}{\pi} \sum_{n=0}^{\infty} \frac{k H_{n}^{(2) \prime}(k R)}{H_{n}^{(2)}(k R)} \int_{0}^{2 \pi} \cos n\left(\theta-\theta^{\prime}\right) u\left(R, \theta^{\prime}\right) \mathrm{d} \theta^{\prime},
$$

where $H_{n}^{(2)}(\bullet)$ is the $n$-th order Hankel function of the second kind; $H_{n}^{(2) \prime}(\bullet)$ is the derivative of this function with respect to its argument. The prime (I) after the sum indicates that the term with $n=0$ is multiplied by $1 / 2$. The condition is exact for an infinite number of terms in the summation. In practice, the series will be truncated to $n_{\max }$ terms, yielding an approximated condition $M^{n_{\max }}$ based on the harmonics up to the truncation number $n_{\max }$. Harari and Hughes [27] proved that choosing $n_{\max }>k R$ guarantees the uniqueness of the solution. This condition can be relaxed, however, when using the modified DtN formulation [7] (MDtN). The idea of this modification is to apply a simple, approximating non-reflecting boundary condition to all harmonics not included in (9). Such a condition can be written as:

$$
\frac{\partial u(R, \theta)}{\partial r}=B u
$$

with $B$ an appropriate constant. A common selection is $B=j k$, such that (10) approximates the Sommerfeld radiation condition (3). The modified DtN condition is then given as:

$$
\frac{\partial u(R, \theta)}{\partial r}=\left(M^{n_{\max }}-B^{n_{\max }}\right) u+B u
$$

with the operators $M^{n_{\max }}$ and $B^{n_{\max }}$ defined as:

$$
\begin{aligned}
M^{n_{\max }} u & =\frac{1}{\pi} \sum_{n=0}^{n_{\max }}, \frac{k H_{n}^{(2) \prime}(k R)}{H_{n}^{(2)}(k R)} \int_{0}^{2 \pi} \cos n\left(\theta-\theta^{\prime}\right) u\left(R, \theta^{\prime}\right) \mathrm{d} \theta^{\prime}, \\
B^{n_{\max }} u & =\frac{1}{\pi} \sum_{n=0}^{n_{\max }} B \int_{0}^{2 \pi} \cos n\left(\theta-\theta^{\prime}\right) u\left(R, \theta^{\prime}\right) \mathrm{d} \theta^{\prime} .
\end{aligned}
$$


The Neumann data resulting from the DtN map is enforced on the FE model over $\Gamma_{t}$. The operator $\mathcal{T}(6)$ can now be derived from the DtN map. Based on (12) and (13), the boundary operator $\mathcal{T}$ for the DtN map can be defined as:

$$
\begin{array}{ll}
\mathcal{T}(u)=M^{n_{\max }} u & \text { for the standard DtN }, \\
\mathcal{T}(u)=\left(M^{n_{\max }}-B^{n_{\max }}\right) u+B u & \text { for the modified DtN. }
\end{array}
$$

The FE system (7) can be completed using these derived expressions for $\mathcal{T}$ : substituting the DtN condition (14) in the weak form yields a matrix system in the FE unknowns $\boldsymbol{d}$ :

$$
\left[\boldsymbol{A}_{F E}+\boldsymbol{A}_{D t N}\right][\boldsymbol{d}]=\left[\boldsymbol{f}_{F E}\right] .
$$

The matrices $\boldsymbol{A}_{F E}$ and $\boldsymbol{f}_{F E}$ result from integrating the residuals on the Helmholtz equation and the boundary conditions, respectively:

$$
\begin{gathered}
\boldsymbol{A}_{F E}=\int_{\Omega_{b}}\left(\nabla u \cdot \nabla v-k^{2} u v\right) \mathrm{d} \Omega_{b}, \\
\boldsymbol{f}_{F E}=-\int_{\Gamma_{b}} v \mathcal{B}(u) \mathrm{d} \Gamma_{b}-\int_{\Omega_{b}}(\mathcal{F} v) \mathrm{d} \Omega_{b} .
\end{gathered}
$$

The vector $\boldsymbol{f}_{F E}$ describes the effect of acoustic sources present in the problem domain. Besides, it consists only of known components in the case of a Neumann boundary condition. For Robin conditions, equation (17) will also yield a contribution to the FE system in the left-hand side.

The matrix $\boldsymbol{A}_{D t N}$ results from integrating the boundary operator $\mathcal{T}$, in this case resulting from the DtN map, over $\Gamma_{t}$ :

$$
\begin{aligned}
\boldsymbol{A}_{D t N} & =\int_{\Gamma_{t}} v \mathcal{T} \mathrm{d} \Gamma_{t}=\int_{\Gamma_{t}} v\left[\left(M^{n_{\max }}-B^{n_{\max }}\right) u+B u\right] \mathrm{d} \Gamma_{t} \\
& =\int_{\Gamma_{t}} v\left(M^{n_{\max }}-B^{n_{\max }}\right) u \mathrm{~d} \Gamma_{t}+\int_{\Gamma_{t}} v B u \mathrm{~d} \Gamma_{t} .
\end{aligned}
$$


When evaluated with the definition of $M$ and $B$ from equation (9) and (10) respectively, and limiting $(M-B)$ to the first $n_{\max }$ circumferential modes on $\Gamma_{t}$, this yields:

$$
\begin{aligned}
\boldsymbol{A}_{D t N} & =\int_{\Gamma_{t}} v \frac{1}{\pi} \sum_{n=0}^{n_{\max }} '\left[\frac{k H_{n}^{(2) \prime}(k R)}{H_{n}^{(2)}(k R)}-j k\right] \int_{0}^{2 \pi} \cos n\left(\theta-\theta^{\prime}\right) u\left(R, \theta^{\prime}\right) \mathrm{d} \theta^{\prime} \mathrm{d} \theta \\
& +\int_{\Gamma_{t}} v j k u \mathrm{~d} \Gamma_{t} .
\end{aligned}
$$

As this expression contains nested integrals, the construction of $\boldsymbol{A}_{D t N}$ is relatively expensive. The computational costs and system properties are discussed in detail in section 5 .

\section{Construction of $\mathcal{T}$ : hybrid FE-WB model}

\subsection{Hybrid modelling concept and procedure}

The use of an unbounded WB model in a hybrid FE-WBM allows this resulting hybrid method to inherently tackle unbounded problems. As such, this explicit unbounded WB model can be seen as an alternative to the techniques described in the introduction section, enabling the application of the FEM to unbounded problems. It shares many similarities with the DtN FE model, using a similar expansion of analytic solutions to describe the field in the unbounded region exterior to $\Gamma_{t}$. Contrarily to the DtN method, in which this expansion is never constructed explicitly, but is instead used to derive a mapping between Dirichlet and Neumann data on $\Gamma$, additional degrees of freedom are introduced in the FE-WBM method to account for the unbounded region of the problem domain.

In an unbounded hybrid FE-WB model, both the FEM and the WBM will be applied in two geometrically non-overlapping regions of the problem. 
The FE domain is confined to the bounded region $\Omega_{b}$ as illustrated in figure 2, similarly as for the DtN FE model. The WBM is used to explicitly describe the field in the unbounded region $\Omega_{u b}$ exterior to $\Gamma_{t}$.

Both the FE and WB submodels are constructed following the usual procedures for both methods. The FE system matrices are obtained by integration of the weak form of the Helmholtz equation and the (natural) boundary conditions (7). The WB system results from enforcing the boundary and interface conditions as explained below; in the case of an unbounded WB model as considered here, the only conditions enforced on the WB model are the interface conditions on $\Gamma_{t}$, imposing continuity with the FE solution in $\Omega_{b}$.

\subsection{An explicit $W B$ model}

The WBM [15] is a numerical modelling method based on an indirect Trefftz approach: the dynamic field is described using an expansion of wave functions, which exactly satisfy the governing equations. The degrees of freedom are the weighting factors of the wave functions in this expansion. The WBM has been applied for unbounded Helmholtz problems, and has proven to be an efficient alternative to element based techniques [21, 22]. Also, a coupling between a FE and a WB model for solving interior acoustic problems has been successfully examined [25], indicating the possibility to combine both the flexibility of the FEM and the high computational efficiency of the WBM in a hybrid method. The use of an unbounded WB model in such a hybrid approach, allows to construct an efficient explicit model for the unbounded domain $\Omega_{u b}$ exterior to the truncation $\Gamma_{t}$. 


\subsubsection{Expansion of the dynamic field}

The dynamic field $p(\boldsymbol{r})$ in the unbounded region $\Omega_{u b}$ is approximated by a solution expansion $\hat{u}(\boldsymbol{r})$ :

$$
\begin{aligned}
p(\boldsymbol{r}) \simeq \hat{u}(\boldsymbol{r}) & =\sum_{w=1}^{n_{w}} \hat{d}_{w} \Phi_{w}(\boldsymbol{r})+\hat{u}_{f}(\boldsymbol{r}) \\
& =\boldsymbol{\Phi} \hat{\boldsymbol{d}}+\hat{u}_{f} .
\end{aligned}
$$

The wave function contributions $\hat{d}_{w}$ are the weighting factors for each of the a priori selected wave functions $\Phi_{w}(\boldsymbol{r})$. The set of all $n_{w}$ wave function contributions $\hat{d}_{w}$ is collected in the column vector $\hat{\boldsymbol{d}}$, while the row vector $\boldsymbol{\Phi}$ contains all $n_{w}$ wave functions. Additionally, $\hat{u}_{f}(\boldsymbol{r})$ represents a particular solution resulting from the acoustic source term $\mathcal{F}(\boldsymbol{r})$ in the right hand side of the inhomogeneous Helmholtz equation (1).

\subsubsection{Wave functions for an unbounded domain}

In addition to being homogeneous solutions of the Helmholtz equation, the wave functions for the unbounded domain are chosen to implicitly satisfy the Sommerfeld radiation condition (3). This removes the need to explicitly impose a radiation condition, similar as in the BEM. Herrera [28] shows that the following expansion $\hat{p}_{e}$, exterior to a circular truncation curve with radius $R$, yields a convergent set for the pressure field $p_{e}$, defined by a Neumann condition on the infinitely long cylinder with radius $R$ :

$$
\begin{aligned}
p_{e}(r, \theta) \simeq \hat{p}_{e}(r, \theta) & =p_{e, c 0} H_{0}^{(2)}(k r) \\
& +\sum_{n=1}^{n_{u}}\left(p_{e, c n} H_{n}^{(2)}(k r) \cos (n \theta)+p_{e, s n} H_{n}^{(2)}(k r) \sin (n \theta)\right),
\end{aligned}
$$


where the contributions $p_{e, c 0}, p_{e, c n}$ and $p_{e, s n}$ are determined by the velocity boundary condition. From this expansion, the following wave function set for unbounded domains is derived [29]:

$$
\Phi_{w}(\boldsymbol{r}(r, \theta))=\left\{\begin{array}{c}
\Phi_{w_{c}}(r, \theta)=H_{w}^{(2)}(k r) \cos (w \theta) \\
\Phi_{w_{s}}(r, \theta)=H_{w}^{(2)}(k r) \sin (w \theta)
\end{array} .\right.
$$

For unbounded problems a commonly used excitation for scattering problems is a plane wave source. For this source, the particular term in solution expansion (20) yields:

$$
\hat{u}_{f}(x, y)=Q_{p w} e^{j k\left(\boldsymbol{d}\left(r_{q}\right)\right)}
$$

with $Q_{p w}$ the plane wave amplitude, $r_{q}=\sqrt{\left(x-x_{q}\right)^{2}+\left(y-y_{q}\right)^{2}}$ and $\boldsymbol{d}\left(r_{q}\right)$ the propagation vector.

\subsection{Hybrid coupling conditions}

The WB system of equation is constructed by enforcing the boundary and continuity conditions through the weighted residual formulation. In this case, where the WBM is used in a hybrid FE-WB model, the only conditions that are imposed on the WB model are the continuity conditions with the FE model on $\Gamma_{t}$ as the wave functions inherently fulfill the Helmholtz (1) and the Sommerfeld radiation condition (3). If the residual presenting this condition is defined as $R_{I}^{W B, F E}$, the WB weighted residual formulation can be written as:

$$
\int_{\Gamma_{t}} \nabla \tilde{p} \cdot \boldsymbol{n} R_{I}^{W B, F E} \mathrm{~d} \Gamma_{t}=0
$$

A direct pressure and velocity coupling is used, imposing pressure continuity 


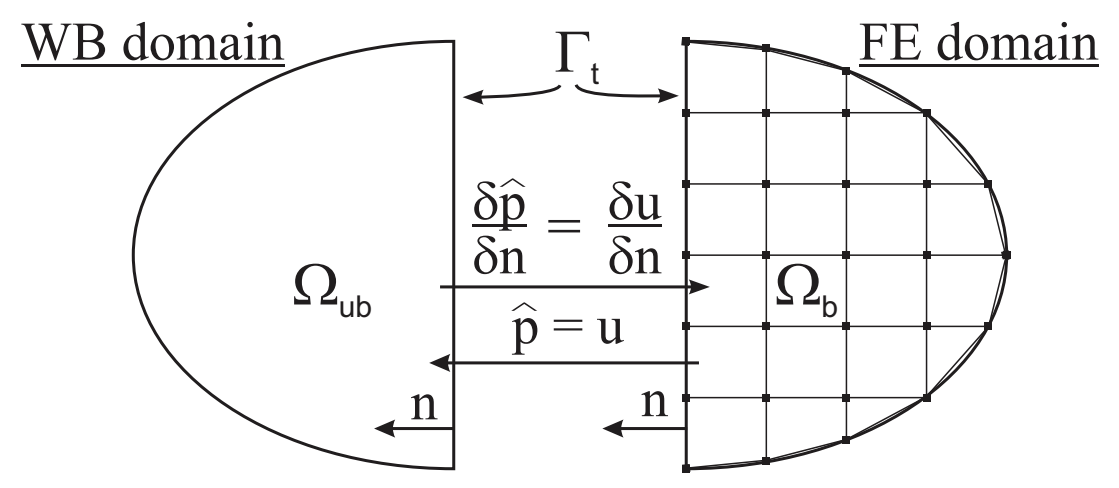

Figure 3: Illustration of the hybrid coupling between the FE and WB models

on the WB model, and velocity continuity on the FEM part, as illustrated in figure 3. The residuals for this coupling can be written as:

$$
\begin{aligned}
& R_{I, p}^{W B, F E}=\hat{p}-u, \\
& R_{I, v}^{F E, W B}=(\nabla u-\nabla \hat{p}) \cdot \boldsymbol{n} .
\end{aligned}
$$

the normal $\boldsymbol{n}$ is the outwards pointing normal on $\Gamma_{t}$. Van Hal [30] and Pluymers [31] discuss alternative coupling conditions for hybrid models. Both direct and indirect couplings, based on additional frame variables, are considered. In this study, the direct pressure-velocity coupling is used, due to its similarity with the DtN formulation: both the unbounded WBM and the DtN take pressure data on $\Gamma_{t}$ as input, whereas velocity data is coupled back to the FE model.

Using those residuals, the WB weighted residual formulation (24) can be completed. Evaluating the WB residual formulation using the pressure continuity on the WB model yields:

$$
\int_{\Gamma_{t}} \nabla \tilde{p} \cdot \boldsymbol{n}(\hat{p}-u) \mathrm{d} \Gamma_{t}=0
$$


The test functions $\tilde{p}$ are expanded using the same wave functions as for the pressure expansion, following a Galerkin approach:

$$
\tilde{p}=\sum_{w=1}^{n_{w}} \tilde{p}_{w} \Phi_{w}=\boldsymbol{\Phi} \tilde{\boldsymbol{p}}_{\boldsymbol{w}}
$$

Equation (27) imposes the only conditions present on the WB model, and consequently constitutes the WB system matrix.

The back-coupling to the FE model is achieved by imposing velocity continuity between the WBM and FEM on the truncation, using the residual (26). This yields the following term in the FE part of the weighted residual formulation:

$$
\int_{\Gamma_{t}} v(\nabla \hat{p}) \cdot \boldsymbol{n} \mathrm{d} \Gamma_{t}
$$

From this expression, the formulation of the boundary operator $\mathcal{T}$, see equation (6) can be determined as:

$$
\mathcal{T}=\nabla \hat{p} \cdot \boldsymbol{n}
$$

\subsection{Resulting hybrid system}

Substituting the operator $\mathcal{T}$ associated with the velocity continuity condition in (7) and introducing the WB field variable expansion (22) completes the system of equations for the FE part of the problem. Complemented with the WB model (24), a coupled system in the FE $(\boldsymbol{d})$ and WB $\left(\boldsymbol{p}_{\boldsymbol{w}}\right)$ unknowns is obtained:

$$
\left[\begin{array}{cc}
\boldsymbol{A}_{F E} & \boldsymbol{C}_{F E-W B} \\
\boldsymbol{C}_{W B-F E} & \boldsymbol{A}_{W B}
\end{array}\right]\left[\begin{array}{c}
\boldsymbol{d} \\
\boldsymbol{p}_{\boldsymbol{w}}
\end{array}\right]=\left[\begin{array}{c}
\boldsymbol{f}_{F E}+\hat{\boldsymbol{f}}_{F E} \\
\hat{\boldsymbol{f}}_{W B}
\end{array}\right]
$$

The matrices $\boldsymbol{A}_{F E}$ and $\boldsymbol{f}_{F E}$ result from integrating the residuals on the Helmholtz equation and the boundary conditions, respectively, and are iden- 
tical to the matrices used in the DtN FE model, given by equations (16) and (17).

The matrix $\boldsymbol{C}_{F E-W B}$ results from integrating $\mathcal{T}$, representing the velocity continuity applied on the FE model:

$$
\boldsymbol{C}_{F E-W B}=\int_{\Gamma_{t}} v \mathcal{T}(u) d \Gamma_{t}=\int_{\Gamma_{t}} v \nabla \hat{p} \cdot \boldsymbol{n} \mathrm{d} \Gamma_{t} .
$$

$\boldsymbol{C}_{W B-F E}$ and $\boldsymbol{A}_{W B}$ are obtained from evaluating the pressure continuity on the WB model:

$$
\begin{gathered}
\boldsymbol{C}_{W B-F E}=-\int_{\Gamma_{t}} \nabla \tilde{p} \cdot \boldsymbol{n} u \mathrm{~d} \Gamma_{t}, \\
\boldsymbol{A}_{W B}=\int_{\Gamma_{t}} \nabla \tilde{p} \cdot \boldsymbol{n} \hat{p} \mathrm{~d} \Gamma_{t} .
\end{gathered}
$$

The loading vectors $\hat{\boldsymbol{f}}_{F E}$ and $\hat{\boldsymbol{f}}_{W B}$ result from the possible presence of a particular solution $\hat{\boldsymbol{f}}$ in the WB pressure expansion (20).

On the truncation circle $\Gamma_{t}$, all WB DOFs and FE DOFs on $\Gamma_{t}$ are coupled together. This results in coupling matrices $\mathbf{C}_{F E-W B}$ and $\mathbf{C}_{W B-F E}$ which may be either dense or sparsely populated without a banded structure. As a result, the rows and columns for the entire system of equations (31) cannot be reordered such that the sparsity of the dynamic stiffness matrix $\boldsymbol{A}_{F E}$ can be exploited when solving the full coupled model at once. To make optimal use of the properties of the submatrices in equation (31) Van Hal [25] and Pluymers [31] propose a three-step solution sequence for a general hybrid FE-WB numerical model of form (31):

1. In a first step, the FE DOFs are eliminated from the system of equations (31) by using the top equation to write the FEM DOFs $\boldsymbol{d}$ as a function of the wave function contributions $\boldsymbol{p}_{\boldsymbol{w}}$ :

$$
\mathbf{d}=\mathbf{A}_{F E}^{-1}\left(\boldsymbol{f}_{F E}+\hat{\boldsymbol{f}}_{F E}-\mathbf{C}_{F E-W B} \boldsymbol{p}_{\boldsymbol{w}}\right),
$$


where $\bullet^{-1}$ represents the inverse of a square matrix. Introduction of (35) in the bottom equation of (31) and regrouping of the terms results in:

$$
\left(\mathbf{A}_{W B}-\mathbf{C}_{W B-F E} \mathbf{H}\right) \boldsymbol{p}_{\boldsymbol{w}}=\hat{\boldsymbol{f}}_{W B}-\mathbf{C}_{W B-F E} \boldsymbol{h} .
$$

The $\left(n_{f e} \times n_{w}\right)$ matrix $\mathbf{H}$ and $\left(n_{f e} \times 1\right)$ vector $\mathbf{h}$ are obtained by solution of two sparse systems of algebraic equations:

$$
\mathbf{A}_{F E} \mathbf{H}=\mathbf{C}_{F E-W B}, \quad \mathbf{A}_{F E} \mathbf{h}=\boldsymbol{f}_{F E}+\hat{\boldsymbol{f}}_{F E} .
$$

Since the coefficient matrix of both systems of equations in (37) is the uncoupled structural FE dynamic stiffness matrix, highly optimised sparse solution algorithms can be employed to obtain the matrices $\mathbf{H}$ and $\mathbf{h}$. This is done by solving the system once with multiple right-hand sides.

2. Next, the remaining dense system of equations (36) is solved using a dense solution algorithm to obtain the wave function contributions $\boldsymbol{p}_{\boldsymbol{w}}$.

3. Finally, the FEM DOFs $\boldsymbol{d}$ are retrieved through a simple matrix multiplication:

$$
\mathbf{d}=-\mathbf{H p}_{\mathbf{w}}+\mathbf{h}
$$

\section{System properties comparison}

This section compares the properties of the DtN map with those of the hybrid FE-WBM presented in the previous section. 


\subsection{Accuracy}

Both the DtN map and the explicit WB model use the same analytical formulation for the dynamic field outside of $\Gamma_{t}$ : a product of harmonics on the circumference of the truncation and Hankel functions, describing the radial decay of the outgoing waves. When using the same truncation order in the series, both methods are expected to deliver equally accurate results.

\subsection{System construction}

The FE matrices $\boldsymbol{A}_{F E}$ and $\boldsymbol{f}_{F E}$ are common to both approaches, as indicated before. They are frequency-independent and relatively easy to construct, requiring only integration of polynomial functions. Those integrals are evaluated numerically using a standard Gauss quadrature rule [32]. In this way, an integral of the form

$$
\int_{\Omega} g(\boldsymbol{v}, \boldsymbol{x}) f(\boldsymbol{u}, \boldsymbol{x}) \mathrm{d} \boldsymbol{x} \approx \sum_{i=1}^{n_{g p}} w_{i} g\left(\boldsymbol{v}, x_{i}\right) f\left(\boldsymbol{u}, x_{i}\right)
$$

with $f(v, x)$ and $g(u, x)$ linear operators, can be evaluated with a computational cost $t_{\text {int }}$ proportional to the size of the involved vectors $\boldsymbol{u}$ and $\boldsymbol{v}$ and the number of integration points $n_{g p}$ :

$$
t_{i n t} \sim \operatorname{size}(\boldsymbol{u}) n_{g p} \operatorname{size}(\boldsymbol{v})
$$

In this relation the computational time is assumed to scale linearly with the number of basic operations (floating point operations or FLOPs) required to complete the computation.

In the case of $\mathrm{FE}$ or WB system matrix construction, the form (39) can be specified as:

$$
\sum_{i=1}^{n_{g p}} w_{i} g\left(\boldsymbol{v}, x_{i}\right) f\left(\boldsymbol{u}, x_{i}\right)=\sum_{i=1}^{n_{g p}} w_{i} g\left(N_{p, i}\left(x_{i}\right) \boldsymbol{v}\right) f\left(N_{p, i}\left(x_{i}\right) \boldsymbol{u}\right),
$$


with $\boldsymbol{u}$ and $\boldsymbol{v}$ the vectors gathering the DOFs and test function weightings, respectively; $N_{p, i}$ represent some general shape functions relating the DOFs to the dynamic field.

$\boldsymbol{D t N} \boldsymbol{F E M}$. In the case of the DtN formulation, all additional effort is spent constructing and integrating the DtN operator (9), to obtain the DtN contribution matrix $\boldsymbol{A}_{D t N}$ (18). This involves the evaluation of $n_{\max }$ integrals over $\Gamma_{t}$ to obtain the Neumann data on $\Gamma_{t}$ (see eq. (9)). Subsequently, this data is integrated in the weighted residual formulation, requiring another integration over $\Gamma_{t}$. The total time for this operation can be approximated as:

$$
t_{D t N} \sim n_{\max } O\left(n_{g p}^{2} n_{f e, \Gamma_{t}}\right)+O\left(n_{g p} n_{f e, \Gamma_{t}}^{2}\right)+O\left(n_{f e, \Gamma_{t}} n_{g p}\right),
$$

where the first term pertains to the construction of the DtN operator through evaluation of equation $(9)$, with $\operatorname{size}(\boldsymbol{v})=\operatorname{size}(\theta)=n_{g p}$ and $\operatorname{size}(\boldsymbol{u})=$ $n_{f e, \Gamma_{t}}$; this integration is repeated $n_{\max }$ times. The second term relates to the integration of this DtN operator in the FE weak form. Additional time is spent evaluating the trigonometric and Hankel functions, proportional to the number of evaluations. $n_{f e, \Gamma_{t}}$ refers to the number of FE DOFs on $\Gamma_{t}$ : those DOFs are directly involved with the evaluation of the dynamic field on $\Gamma_{t}$ and consequently with the construction and integration of the DtN map. The number of integration points $n_{g p}$ is proportional to $n_{f e, \Gamma_{t}}$ if a fixed number of integration points per element is chosen, as is usual in FE implementations. The total cost of the construction of $\boldsymbol{A}_{D t N}$ is then approximated as:

$$
t_{D t N} \sim O\left(n_{\max } n_{f e, \Gamma_{t}}^{3}\right)
$$


hybrid $\boldsymbol{F E}-\boldsymbol{W B} \boldsymbol{M}$. Construction of the coupled FE-WB model involves the evaluation of three additional integrals in the weighted residual formulation, yielding the matrices $\boldsymbol{C}_{F E-W B}, \boldsymbol{C}_{W B-F E}$ and $\boldsymbol{A}_{W B}$. In this case, however, the construction of the integrands requires only the evaluation of the dynamic field and the first-order derivative from both the FE and WB formulations. No nested integration is required in this case. The cost for the evaluation of those additional integrals is:

$$
\begin{aligned}
t_{F E-W B} & \sim O\left(n_{w}^{2} n_{g p}\right)+2 O\left(n_{w} n_{g p} n_{f e, \Gamma_{t}}\right)+O\left(n_{w} n_{g p}\right) \\
& \approx O\left(n_{\text {max }}^{2} n_{f e, \Gamma_{t}}\right)+O\left(n_{\max } n_{f e, \Gamma_{t}}^{2}\right),
\end{aligned}
$$

with $n_{w} \sim n_{\max }$.

From this short analysis, it is clear that the evaluation of the DtN matrix $\boldsymbol{A}_{D t N}$ is more expensive than the combined coupling/WB system matrices needed in the hybrid FE-WB. This cost is mainly attributed to the expensive evaluation of the DtN operator itself, requiring a summation of integrals over $\Gamma_{t}$. The cost of the DtN formulation rises more quickly as the size of the FE system, and consequently also the number of $\mathrm{FE}$ dofs on $\Gamma_{t}$, increases. Moreover, $n_{f e, \Gamma_{t}}$ will generally be much larger than $n_{\max }$, as illustrated in the numerical verification examples.

\subsection{System solving}

The resulting systems (15) and (31) have substantially different properties, requiring a different approach for solving. The system resulting from the DtN formulation constitutes both the basic FE system, which is in general sparse and often real, and added terms from the DtN condition contained in $\boldsymbol{A}_{D t N}$. Since the DtN map requires integration over the entire truncation, 
this matrix contains equations linking together all DOFs on the truncation. Consequently, the resulting system matrix will partially lose its sparseness, depending on the constitution of the mesh, in particular at the truncation. Additionally, the terms introduced by the DtN condition will be complex. The system can be solved using an optimised FE solver, but the loss in sparsity and the added complex terms may deteriorate the efficiency of these algorithms.

The coupled FE-WB system is best solved according to the procedure detailed above. This involves the solution of the original FE system matrix $\boldsymbol{A}_{F E}$ with multiple (complex) right-hand sides to obtain $\boldsymbol{H}$ and $\boldsymbol{h}$. Since this matrix retains its real and sparse nature, it is very suitable for efficient sparse solvers, in contrast to the system obtained using the DtN method. Additionally, a small but complex and dense system needs to be solved to obtain the WB DOFs. This system is usually much smaller than the FE system $\left(n_{w}<<n_{f e}\right)$, resulting in a small additional computational effort.

\section{Numerical examples}

This section discusses two two-dimensional numerical examples. In the first example, the DtN map (modified formulation) and the explicit WB model are evaluated for the case of a rigid cylinder scattering an incident plane wave. For this problem, an analytical reference solution is available. A second example considers the scattering of a star-shaped non-convex polygon.

In both cases, the aim is to investigate the relative performance of both methods in their ability as an ABC, allowing an FE model to solve an unbounded problem. To this end, both methods are implemented based on 
a common FE framework. Matlab R2009a is used as software platform for constructing the system matrices; the system solution (direct solution for the DtN and three-step procedure for the FE-WB) is computed using MD.Nastran R3. All calculations are performed on an intel Xeon 5540 based system with 24 GB memory running a linux operating system.

\subsection{Scattering of a rigid cylinder}

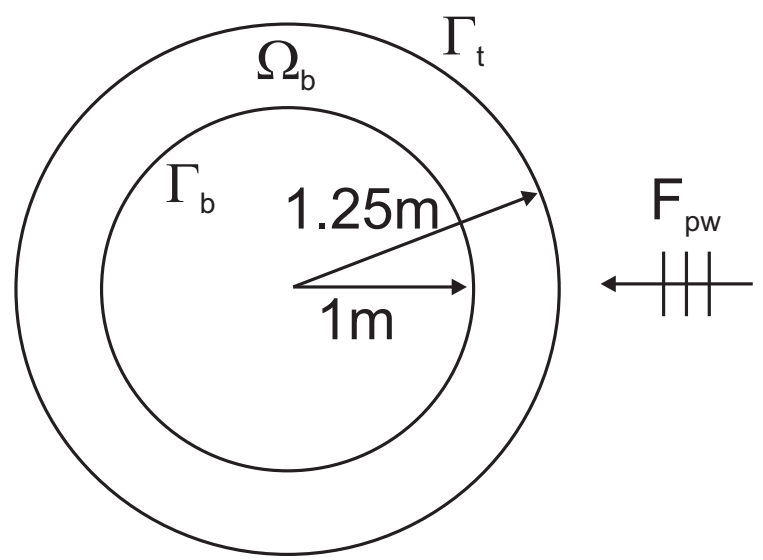

Figure 4: Geometry and source definition

The scattering of a plane wave, incident along the negative $\mathrm{X}$-axis on a rigid circle (cylinder) with radius $1 \mathrm{~m}$ is considered. The acoustic fluid is air $\left(c=340 \mathrm{~m} / \mathrm{s}, \rho_{0}=1.225 \mathrm{~kg} / \mathrm{m}^{3}\right)$. This simple geometry is chosen due to the availability of a reference solution for this problem, which can be written in the form of a series expansion [33]:

$$
p_{a}(r, \theta)=-2 \sum_{n}^{\prime} j^{n} \frac{J_{n}^{\prime}(k a)}{H_{n}^{(2) \prime}(k a)} H_{n}^{(2)}(k r) \cos (n \theta),
$$

with $(r, \theta)$ the polar coordinates of the observation point and $J_{n}$ is the ordinary Bessel function of the first kind and order $n$. This analytical solution 
provides a good reference for the evaluation of the accuracy of the different approaches.

Several linear FE models are constructed for the problem, with mesh size $h$ varying between $50 \mathrm{~mm}$ and $7 \mathrm{~mm}$. The details, including an estimation of the mesh validity, are given in table 1 . The FE model covers the bounded region $\Omega_{b}$ of the problem, as indicated in figure 4 . As a measure of the accuracy, the $\mathrm{L}^{2}$ norm relative error is used:

$$
\mathrm{L}^{2} \text { norm relative error }=\left(\frac{\int_{\Omega}\left|u_{r e f}-u\right|^{2} d \Omega}{\int_{\Omega}\left|u_{r e f}\right|^{2} \mathrm{~d} \Omega}\right)^{1 / 2} .
$$

\begin{tabular}{r|cccc}
\hline max. mesh size $[\mathrm{mm}]$ & 50 & 25 & 12 & 7 \\
\hline \# DOFs & 2068 & 7917 & 30914 & 85476 \\
$f_{\max }^{*}: 6 / \lambda$ & 1130 & 2265 & 4530 & 7550 \\
$f_{\max }^{*}:$ pollution & 399 & 633 & 1032 & 1478 \\
\hline
\end{tabular}

Table 1: FE model information for the cylinder model. * maximum frequency considering interpolation (6 elements per wavelength) and pollution errors [34]

Figure 5 illustrates the amplitude of the scattered field at $2000 \mathrm{~Hz}(k a \approx$ $40 \pi$ ), calculated using a hybrid FE-WB model. In this case, the $7 \mathrm{~mm}$ mesh is used with 50 orders in the unbounded model, yielding a very good error of $0.03 \%$ relative to the analytical solution over the computational domain $\Omega_{b}$.

To assess the efficiency of the different approaches, a convergence study is carried out using the meshes described in table 1 and for increasing number of orders in both the MDtN map and the unbounded WB model. The $\mathrm{L}^{2}$ norm relative error is used as a measure of the accuracy. Figure 6 shows 


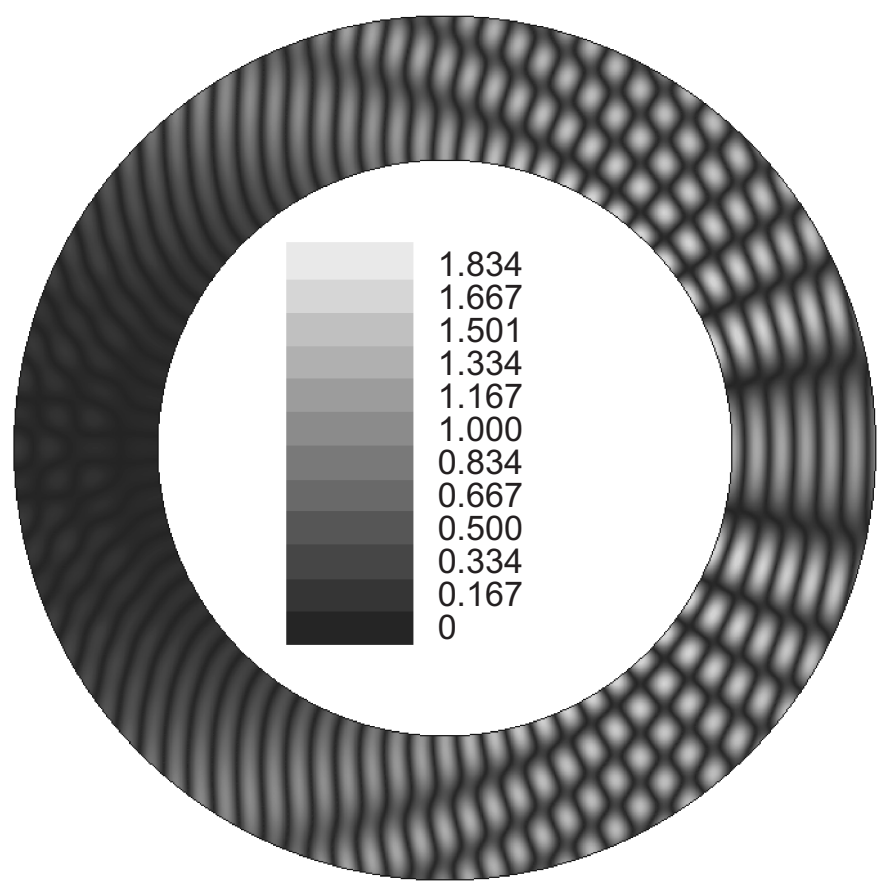

Figure 5: Amplitude of the scattered field, $2000 \mathrm{~Hz}(k a \approx 40 \pi)$

the convergence results for different wave numbers from $250 \mathrm{~Hz}(k a \approx 5 \pi)$ to $2000 \mathrm{~Hz}(k a \approx 40 \pi)$, using the different meshes. The number of orders in the unbounded model $n_{\max }$ is increased from 5 to 100 , both for the MDtN and the WB case. For a same number of orders, almost the same level of accuracy is obtained irrespective of the method used. The difference is only due to the application of the approximating non-reflecting boundary condition for the discarded harmonics of the DtN map, see eq. (11). This modification is more effective at higher frequencies as illustrated in figure 7 for the $7 \mathrm{~mm}$ mesh. As mentioned before, this is to be expected since both approaches are based on the same mathematical description of the exterior field. It is clear, however, that the WB implementation yields consistently faster calculations; 
this advantage gets more pronounced as the FE model size grows.
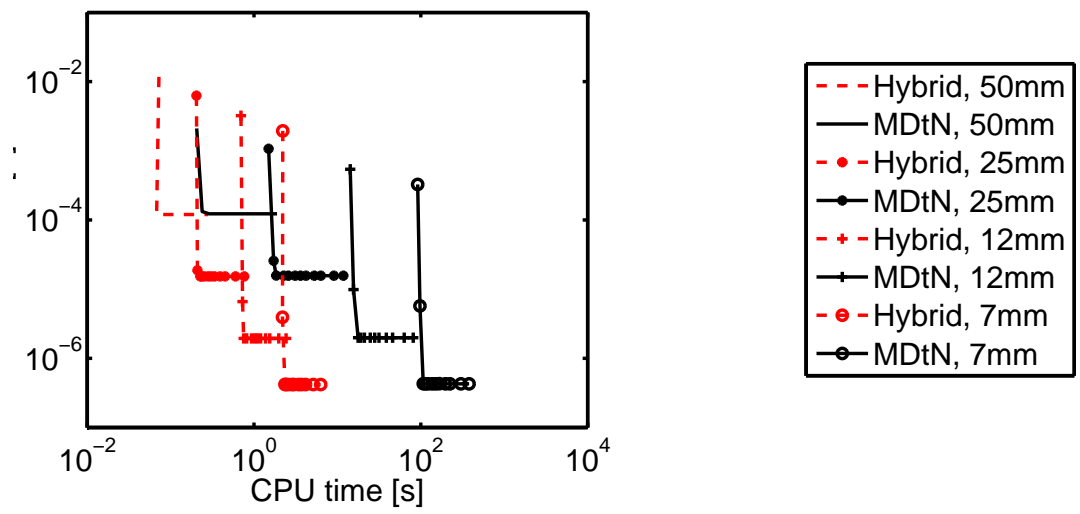

(a) $250 \mathrm{~Hz}(k a \approx 5 \pi)$

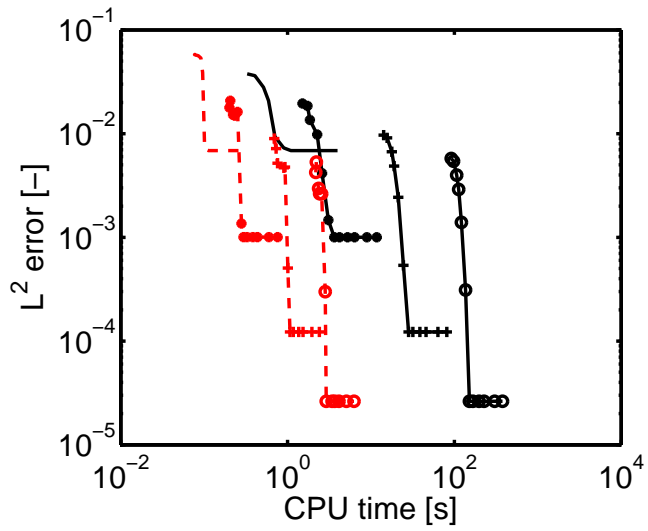

(b) $1000 \mathrm{~Hz}(k a \approx 20 \pi)$

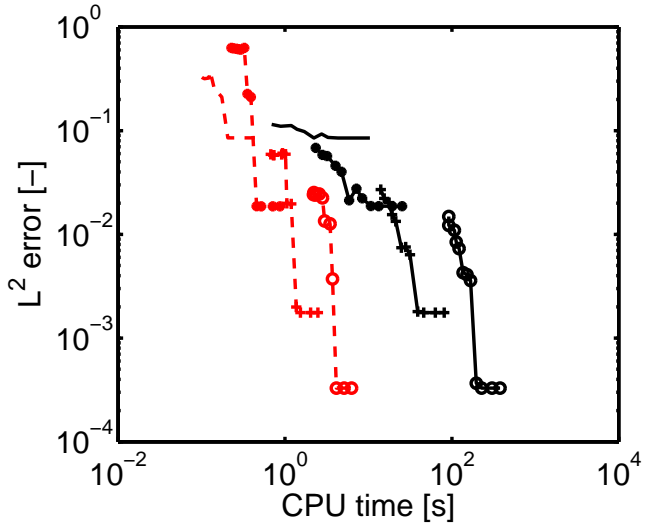

(c) $2000 \mathrm{~Hz}(k a \approx 40 \pi)$

Figure 6: Error convergence for increasing $n_{\max }$, comparison between modified $\mathrm{DtN}$ and WBM

On examining more closely the relative CPU time of the different contributions, listed in table 2 for the $7 \mathrm{~mm}$ mesh, it appears that the main computational advantage of the WBM is in the construction of the operator $\mathcal{T}$ : whereas the construction and integration of the DtN map takes the ma- 

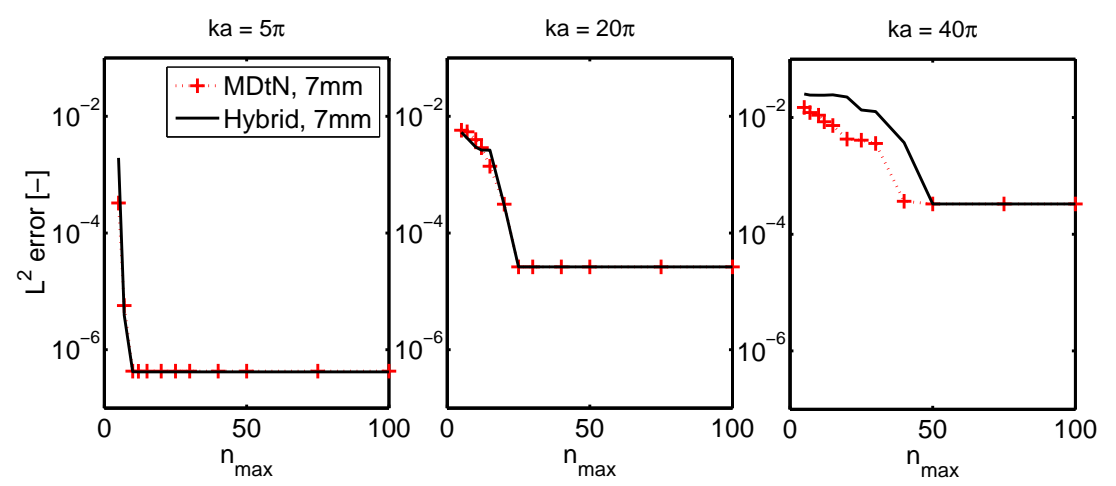

Figure 7: Error convergence for the $7 \mathrm{~mm}$ mesh, shown for increasing $n_{\max }$. Comparison between modified DtN and WBM

jority of the time in the DtN method, the contribution of the WB model and interface construction is far less dominant. The computational advantage of the WBM is clear when comparing the construction time. On average the time for WB system construction is only about $0.5 \%$ of the time needed to construct the equivalent DtN map.

If the solving time is considered, the effectiveness of the three-step solution procedure (detailed in section 4.4) is reflected in the results: in general, a good speedup can be obtained. This advantage is most pronounced at low $n_{\max }$, due to the rising cost of the matrix operations in the three-step procedure as the number of wave functions grows. For the example considered, the 'elbow' points in the convergence (after which the accuracy is limited by the mesh size only) are at $n_{\max }=10,25$ and 50 for $k a \approx 5 \pi, 20 \pi$ and $40 \pi$, respectively, yielding a WB solving time $20-50 \%$ of the equivalent $\mathrm{DtN}$ model. 


\begin{tabular}{c|cc|cc|cc|}
\multicolumn{2}{c}{ WB system } & \multicolumn{2}{c}{ DtN system } & \multicolumn{2}{c}{ rel., WB vs DtN } \\
$n_{\max }$ & construct. & solving & construct. & solving & construct. & solving \\
\hline 5 & 0.38 & 0.9 & 87 & 6.1 & $0.44 \%$ & $15 \%$ \\
10 & 0.46 & 1.1 & 102 & 6.1 & $0.45 \%$ & $18 \%$ \\
25 & 0.72 & 1.8 & 147 & 6.1 & $0.49 \%$ & $30 \%$ \\
50 & 1.27 & 3.1 & 222 & 6.1 & $0.57 \%$ & $51 \%$ \\
100 & 2.33 & 5.6 & 374 & 6.1 & $0.62 \%$ & $92 \%$ \\
\hline
\end{tabular}

Table 2: System construction and solving time [s] for the DtN and FE-WB methods; FE-WB time relative to the respective time for the DtN method [\%]. Results shown are obtained with the $7 \mathrm{~mm}$ mesh

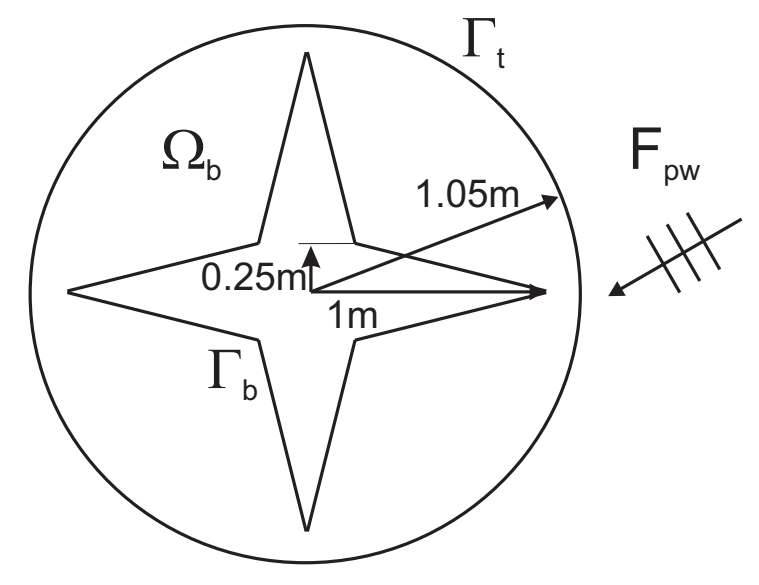

Figure 8: Geometry and source definition

\subsection{Scattering of a non-convex polygon}

A second numerical example studies the scattering of an incident plane wave on a non-convex polygon, as shown in figure 8. The polygon, with corner points measuring $1 \mathrm{~m}$ from the center, is enclosed in a truncation with radius $1.05 \mathrm{~m}$. Rigid boundary conditions are imposed on $\Gamma_{b}$, and the fluid 
properties are taken as air at ambient conditions. The plane wave is incident at an angle of 30 degrees, to obtain an asymmetric excitation.

This more complex geometry gives rise to interference and diffraction patterns in the response, as can be seen in figure 9 showing the amplitude of the dynamic field at $2000 \mathrm{~Hz}$. The pressure field shown is calculated with a hybrid FE-WB model using a $7 \mathrm{~mm}$ mesh and 40 orders in the unbounded domain. Similarly to the previous example, a very good accuracy can be obtained, in this case the $\mathrm{L}^{2}$ norm relative error is $0.5 \%$. The reference for error calculations for this case is obtained from a detailed BEM calculation, using a mesh with element size $0.25 \mathrm{~mm}$.

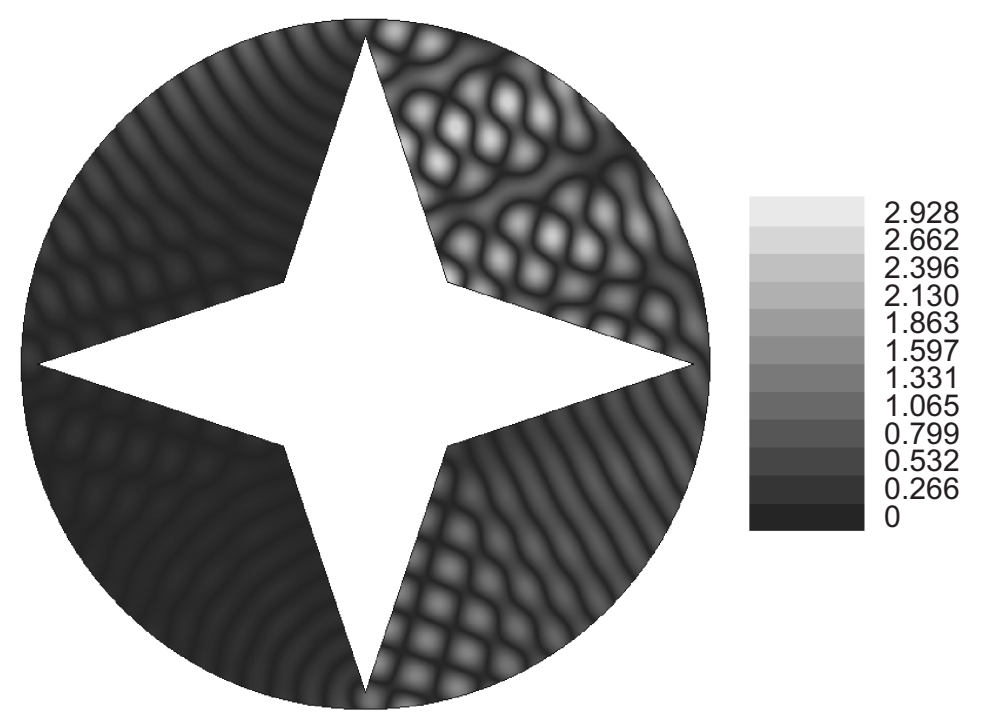

Figure 9: Amplitude of the scattered field, $2000 \mathrm{~Hz}(k a \approx 40 \pi)$

Again, several FE models are constructed to analyse the convergence characteristics of both the DtN and the hybrid FE-WB method. The model details are given in table 3 . The behaviour observed is in general similar to the previous case: for a same value of $n_{\max }$, the same level of accuracy is obtained 


\begin{tabular}{r|cccc}
\hline max. mesh size & 50 & 25 & 12 & 7 \\
\hline$\#$ DOFs & 1884 & 7240 & 28368 & 112288 \\
$f_{\max }^{*}: 6 / \lambda$ & 1125 & 2250 & 4500 & 9000 \\
$f_{\text {max }}^{*}:$ pollution & 399 & 633 & 1032 & 1478 \\
\hline
\end{tabular}

Table 3: FE model information for the polygon model * maximum frequency considering interpolation (6 elements per wavelength) and pollution errors [34]

with the different methods. The computational advantage of the FE-WB method is preserved, as it is based on the more efficient construction of the boundary operator $\mathcal{T}$. In this example, the more complex geometry and the small distance between the boundary and $\Gamma_{t}$ result in short-wavelength waves, often travelling almost tangent to $\Gamma_{t}$. It should be noted that a very good prediction accuracy is obtained, regardless of this more complex dynamic field.

\section{Conclusions}

This paper discusses the use of an explicit unbounded Wave Based (WB) model exterior to the truncation of a FE model of an unbounded problem. The basis functions used in this model are exact solutions of the unbounded Neumann problem exterior to a circle. Therefore, this basis is equivalent to the one used to derive the DtN map for such a truncation. It is shown that this novel approach results in an equivalent accuracy as for the DtN. However, the hybrid FE-WB method framework allows a more efficient construction of the model. Moreover, the structure of the resulting hybrid system allows for a three-step solution where the original FE system is solved with multiple right- 

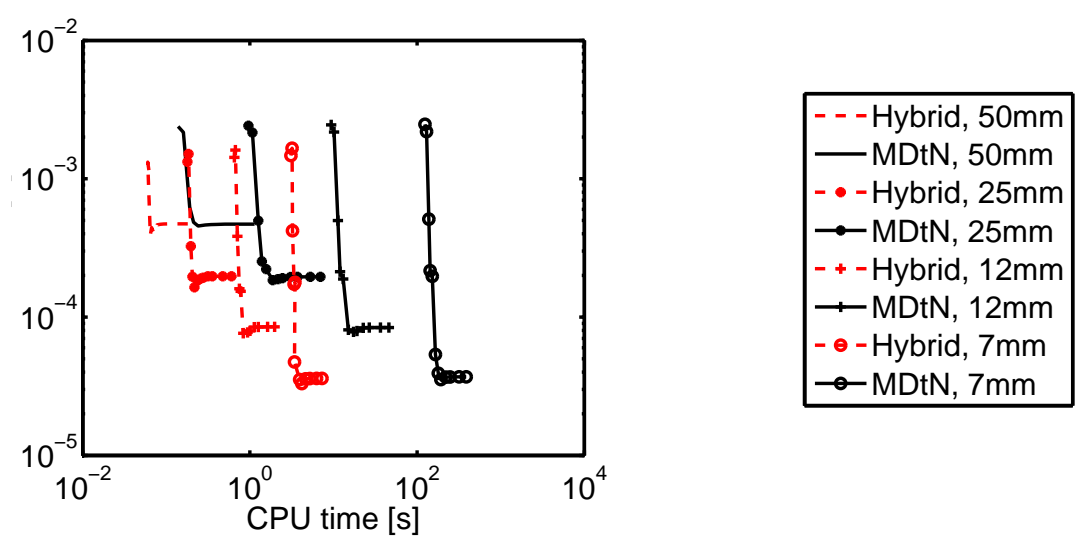

(a) $250 \mathrm{~Hz}(k a \approx 5 \pi)$

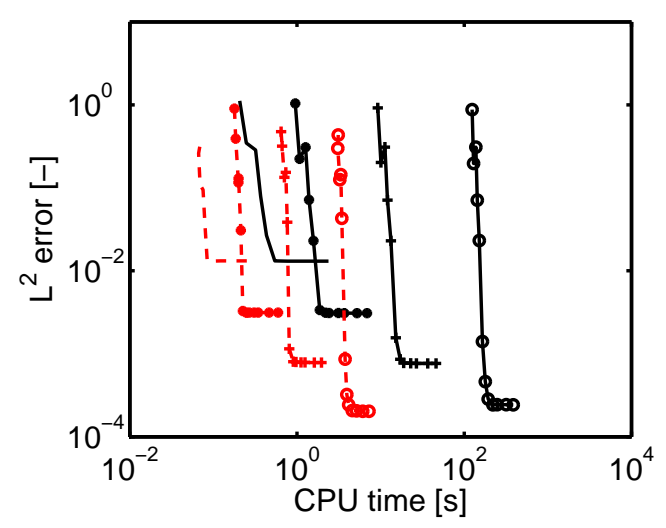

(b) $1000 \mathrm{~Hz}(k a \approx 20 \pi)$

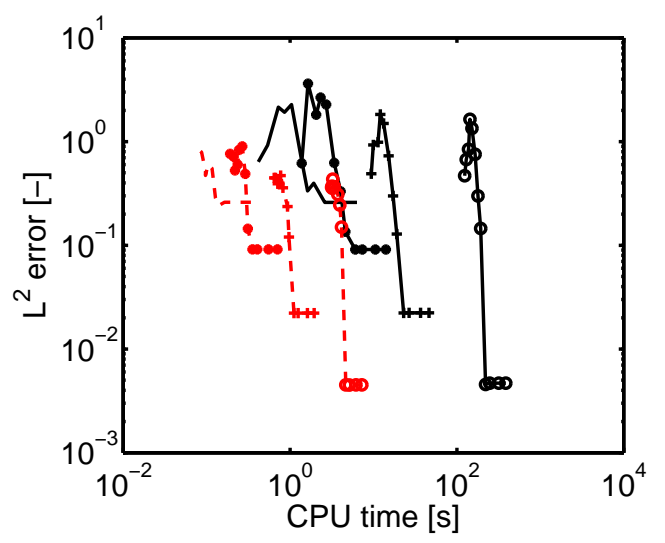

(c) $2000 \mathrm{~Hz}(k a \approx 40 \pi)$

Figure 10: Error convergence for increasing $n_{\max }$, comparison between modified DtN and WBM

hand sides, but the sparse and real matrix structure is retained, allowing the use of efficient FE solution routines. The FE-WB method is validated in two numerical examples, where it is compared to a DtN formulation, illustrating the potential and the computational advantage of the approach. 


\section{Acknowledgements}

The Institute for the Promotion of Innovation through Science and Technology in Flanders (IWT-Vlaanderen) is gratefully acknowledged within the ASTRA project and for the personal grant of Bart Bergen. The Fund for Scientific Research - Flanders (F.W.O.), Belgium is gratefully acknowledged for the personal grants of Elke Deckers and Bert Van Genechten. Besides also the Research Fund KU Leuven is gratefully acknowledged for its support.

\section{References}

[1] L. L. Thompson, A review of finite element methods for time-harmonic acoustics, Journal of the Acoustical Society of America 119 (2006) 13151330.

[2] A. Bayliss, M. Gunzburger, E. Turkel, Boundary Conditions for the Numerical Solution of Elliptic Equations in Exterior Regions, SIAM Journal on Applied Mathematics 42 (1982) 430-451.

[3] B. Engquist, A. Majda, Absorbing boundary conditions for the numerical simulation of waves, Mathematics of Computation 31 (1977) 629-651.

[4] D. Givoli, High-order local non-reflecting boundary conditions: a review, Wave Motion 39 (2004) 319-326.

[5] T. Hagstrom, A. Mar-Or, D. Givoli, High-order local absorbing conditions for the wave equation: Extensions and improvements, Journal of Computational Physics 227 (2008) 3322 - 3357. 
[6] J. B. Keller, D. Givoli, Exact non-reflecting boundary conditions, Journal of Computational Physics 82 (1989) 172-192.

[7] M. Grote, J. Keller, On Nonreflecting Boundary Conditions, Journal of Computational Physics 122 (1995) 231-243.

[8] D. Givoli, Recent advances in the DtN FE method, Archives of Computational Methods in Engineering 6 (1999) 71-116.

[9] J.-P. Berenger, A Perfectly Matched Layer for the Absorption of Electromagnetic Waves, Journal of Computational Physics 114 (1994) 185-200.

[10] F. Collino, P. B. Monk, Optimizing the perfectly matched layer, Computer Methods in Applied Mechanics and Engineering 164 (1998) 157 171.

[11] A. Bermúdez, L. Hervella-Nieto, A. Prieto, R. Rodríguez, Perfectly matched layers for time-harmonic second order elliptic problems, Archives of Computational Methods in Engineering 17 (2010) 77 - 107.

[12] P. Bettess, Infinite Elements, Penshaw Press, 1992.

[13] D. S. Burnett, R. L. Holford, Prolate and oblate spheroidal acoustic infinite elements, Computer Methods in Applied Mechanics and Engineering 158 (1998) 117-141.

[14] R. J. Astley, G. J. Macaulay, J. P. Coyette, Mapped wave envelope elements for acoustical radiation and scattering, Journal of Sound and Vibration 170 (1994) 97-118. 
[15] W. Desmet, A wave based prediction technique for coupled vibroacoustic analysis, KULeuven, division PMA, PhD. thesis 98D12, 1998.

[16] B. Pluymers, B. Van Hal, D. Vandepitte, W. Desmet, Trefftz-based methods for time-harmonic acoustics, Archives of Computational Methods in Engineering (2007) 343-381.

[17] E. Deckers, O. Atak, L. Coox, R. D’Amico, H. Devriendt, S. Jonckheere, K. Koo, B. Pluymers, D. Vandepitte, W. Desmet, The Wave Based Method: an overview of 15 years of research, Wave Motion 51 (2014) $550-565$.

[18] E. Trefftz, Ein Gegenstück zum Ritzschen Verfahren, in: Proceedings of the $2^{\text {nd }}$ International Congress on Applied Mechanics, Zurich, Switzerland, pp. 131-137.

[19] B. Pluymers, W. Desmet, D. Vandepitte, P. Sas, On the Use of a Wave Based Prediction Technique for Steady-State Structural-Acoustic Radiation Analysis, Computer Modeling in Engineering \& Sciences 7 (2005) 173-184.

[20] B. Van Genechten, B. Bergen, D. Vandepitte, W. Desmet, A Trefftzbased numerical modelling framework for Helmholtz problems with complex multiple scatterer configurations, Journal of Computational Physics 229 (2010) 6623-6643.

[21] B. Bergen, B. Van Genechten, D. Vandepitte, W. Desmet, An efficient Trefftz-based method for three-dimensional Helmholtz problems in un- 
bounded domains, Computer Modeling in Engineering \& Sciences 61 (2010) 155-175.

[22] B. Bergen, B. Pluymers, B. Van Genechten, D. Vandepitte, W. Desmet, A Trefftz based method for solving Helmholtz problems in semi-infinite domains, Engineering Analysis with Boundary Elements 36 (2012) 3038.

[23] O. Atak, D. Huybrechs, B. Pluymers, W. Desmet, The design of Helmholtz resonator based acoustic lenses by using the symmetric MultiLevel Wave Based Method and genetic algorithms, Journal of Sound and Vibration 333 (2014) 3367-3381.

[24] B. Van Hal, W. Desmet, D. Vandepitte, P. Sas, Hybrid finite element wave based method for acoustic problems, Computer Assisted Mechanics and Engineering Sciences 11 (2003) 375-390.

[25] B. Van Hal, W. Desmet, D. Vandepitte, Hybrid finite element - wave based method for steady-state interior structural-acoustic problems, Computers \& Structures 83 (2005) 167-180.

[26] D. Colton, R. Kress, Inverse acoustic and electromagnetic scattering theory, Springer-Verlag, Berlin, Heidelbert, New York, 2nd edition, 1998.

[27] I. Harari, T. J. R. Hughes, Analysis of continuous formulations underlying the computation of time-harmonic acoustics in exterior domains, Computer Methods in Applied Mechanics and Engineering 97 (1992) $103-124$. 
[28] I. Herrera, Boundary Methods: an Algebraic Theory, Pitman Adv. Publ. Program, London, 1984.

[29] B. Bergen, Wave Based modelling techniques for unbounded acoustic problems, KULeuven, division PMA, PhD. thesis 2011D07, 2011.

[30] B. Van Hal., Automation and performance optimization of the wave based method for interior structural-acoustic problems, Ph.D. thesis, Katholieke Universiteit Leuven, Departement Werktuigkunde, Leuven, 2004.

[31] B. Pluymers, Wave based modelling methods for steady-state vibroacoustics, KULeuven, division PMA, PhD. thesis 2006D04, 2006.

[32] M. Abramowitz, I. A. Stegun eds., Handbook of Mathematical Functions (with Formulas, Graphs, and Mathematical Tables), Dover Publishers, 1972.

[33] F. Ihlenburg, Finite Element Analysis of Acoustic Scattering, Volume 132 of Applied Mathematical Sciences, Springer, 1998.

[34] P. Bouillard, F. Ihlenburg, Error estimation and adaptivity for the finite element method in acoustics: 2D and 3D applications, Computer Methods in Applied Mechanics and Engineering 176 (1999) 147-163. 